\title{
[Estudios]
}

\section{El respeto por los demás en el marco de la discusión Frankfurt-Raz}

\section{The respect for people in the frame of the Frankfurt-Raz discussion}

\author{
Gabriel Aragón Aranda \\ Universidad de Málaga (España)
}

Recibido: 06.02.2019

Aceptado: 13.01.2020

\section{RESUMEN}

El presente trabajo tiene por objeto estudiar analíticamente la cuestión del respeto atendiendo a su relevancia dentro de la filosofía moral. Para ello nos centraremos en la perspectiva aportada por Harry G. Frankfurt, subsidiaria, en alguna medida, del esclarecimiento previo que realizó a propósito del igualitarismo. Las tesis de Frankfurt serán, posteriormente, contrastadas con el comentario y la disquisición propia que realiza, a propósito del texto de dicho autor, Joseph Raz. Finalmente, tras analizar el diálogo establecido, extraeremos conclusiones respecto al estado de la cuestión y tomaremos posición al respecto dentro de la misma.

PALABRAS CLAVE

RESPETO, IGUALDAD, FRANKFURT, RAZ, MORAL

\section{ABSTRACT}

This paper aims to analytically study the issue of respect focusing on its relevance within the moral philosophy. For this, we will focus on the perspective provided by Harry G. Frankfurt; subsidiary of the prior clarification made on the subject of egalitarianism. The Frankfurt's theses will be, later, contrasted with the commentary and the own disquisition about the issue of Joseph Raz. Finally, after analyzing the established dialogue, we will draw conclusions regarding the state of the matter and take position within it.

KEY WORDS

RESPECT, EQUALITY, FRANKFURT, RAZ, MORAL 
I. INTRODUCCiÓN: El RESPETO A PROPÓSITO DE LA IGUALDAD.

EL PROLEGÓMENo de FrankfurT

Cuando atendemos al eXamen que Realiza Harry G. Frankfurt sobre la noción de respeto, hemos de ser conscientes de que constituye la segunda parte, como manifiestamente se observa, de un ensayo titulado «Igualdad y respeto» ${ }^{1}$. En dicho ensayo, el respeto aflora como una manera de ejemplificar y matizar ciertas cuestiones de lo previamente examinado, a saber, la igualdad.

La primera parte del ensayo, a su vez, enlaza con un ensayo anterior del que podríamos decir que constituye la secuela: «La igualdad como ideal moral $»^{2}$. Así, como si de un hilo lógico se tratase, vamos a tirar del mismo para, a partir de la concepción de Frankfurt sobre la igualdad, ir avanzando hasta la noción de respeto. Con el afán, por tanto, de que esta parte introductoria acerca de la igualdad permita contextualizar el porqué del respeto en toda esta cuestión para, más adelante, entrar a considerarlo de pleno.

Así todo, el propósito primigenio de Frankfurt es realizar una crítica al igualitarismo denunciando la ausencia de cualquier tipo de atributo moral en la idea de igualdad, por tanto que, si es algo, será un medio, no un fin para la ética. Veámoslo un poco más detenidamente.

La cuestión surge a raíz del igualitarismo económico, entendiendo por tal «la doctrina que establece que es deseable que todos tengan la misma cantidad de ingresos y de riquezas ${ }^{3}$, aunque podríamos hablar del igualitarismo en sentido amplio.

Sin embargo, lo realmente importante, moralmente hablando, quedaría acogido por la doctrina de la suficiencia, no por el igualitarismo. Según dicha doctrina, la relevancia moral estaría determinada por el hecho de que todo el mundo tuviese lo suficiente, no lo mismo. Esto no quiere decir que la igualdad no tenga ningún tipo de valor. De hecho, "podría resultar que el enfoque más viable para el logro de la suficiencia fuera la búsqueda

1 Frankfurt, H.G.: «Igualdad y respeto» en Frankfurt, H.G.: Necesidad, volición y amor. Buenos Aires: Katz, 2007.

2 Frankfurt, H.G.: «La igualdad como ideal moral» en Frankfurt, H.G.: La importancia de lo que nos preocupa. Buenos Aires: Katz, 2006.

3 Ibid., p. 195. 
de la igualdad $»^{4}$. Así todo, cualquier tipo de valor moral que pudiéramos achacarle a la igualdad sería, en última instancia, derivado.

Si ha habido alguna obcecación con el atractivo moral que varios filósofos han podido sentir por la igualdad, sería, a ojos de Frankfurt, fruto de una ilusión. Así, ante la reconocida fuerza retórica de la pregunta de Thomas Nagel, a saber, “¿Cómo podría no ser malo que las perspectivas de vida de algunas personas al nacer sean radicalmente inferiores a las de otras?» ${ }^{5}$, Frankfurt replica que, sencillamente, «tener menos es compatible con tener bastante» ${ }^{6}$. Sin embargo, es importante notar que la suficiencia que defiende Frankfurt como moralmente relevante no alude a una suficiencia de mínimos, es decir, a lo que sería entendida como una mera supervivencia. No se trata de convertir nuestra vida en algo meramente tolerable, sino en entender el estar «satisfecho» en otro sentido más amplio: la persona debe de sentirse, en algún aspecto, conforme o feliz con su vida; es decir, no insatisfecha ni angustiada. Lo cual no implica que, por otro lado, la persona no estuviese dispuesta a aceptar un estado mejor de cosas, sino que, simplemente, no va a hacer un esfuerzo proactivo por conseguirlo: se encuentra satisfecha.

Es por ello que la igualdad no representa algo deseable, ya que podría, por supuesto, implicar una igualdad a la baja. La satisfacción personal, particular, por tanto, es lo moralmente relevante: atender a la circunstancia de cada individuo y a lo que necesita o requiere para tener una vida buena.

El caso es que, sin duda, el igualitarismo refiere a una consideración puramente formal del asunto que desprecia a la persona en sí. La doctrina de la suficiencia, por tanto, apela al contenido de la vida que realmente encarna la persona. Así «lo que es de auténtico interés moral no es formal sino sustantivo»?

¿Pero, qué tiene que ver el respeto con todo esto? Es en este punto - de atención a la persona particular - en la que se cuela, de forma subsidiaria, acaso apendicular, la cuestión del respeto.

Dentro del marco de la crítica a la comparación con los demás, vinculada al igualitarismo, Frankfurt establece que sucede de forma similar con el

4 Ibid., p. 197.

5 Como es citado en Frankfurt, H.G.: «Igualdad y respeto» en Frankfurt, H.G.: Necesidad, volición y amor. Buenos Aires: Katz, 2007, p. 231.

6 Ibid., p. 231.

7 Ibid., p. 232. 
respeto. Es decir, se ha de respetar a la persona por lo que ella misma es y no porque haya que respetar a todo el mundo por igual. Así todo, alguien podría sentirse ofendido al estar en una posición inferior a la de otro porque podría pensarse que el responsable de su situación, fuese quien fuese, no lo ha tratado con respeto.

En definitiva, nos encontramos ante un tablero en el que Frankfurt mueve ficha a favor del particularismo, es decir, de atender a la realidad concreta y particular del individuo; que es, en definitiva, ante el que hay que rendir cuentas. Veremos más adelante cómo, por otra parte, esta posición entrará en discusión con la de Raz, la cual podríamos calificar de abstraccionista o universalista.

Mentar la igualdad tenía el propósito de señalar, por parte de Frankfurt, que no estamos ante un elemento moral intrínsecamente valioso y que, en todo caso, su valor moral derivará siempre de otra causa. El paralelismo que se va a establecer con el respeto es precisamente este: no podemos respetar a la humanidad en abstracto, subsumiendo a todos los elementos del conjunto en una categoría universal, sino a los individuos particulares en función de un criterio.

\section{El respeto (I): El enfoque de Frankfurt}

Nos dice Frankfurt que «la diferencia más fundamental entre la igualdad y el respeto tiene que ver con la focalización y la intención» ${ }^{8}$. El respeto no nos dirige a nada externo sino, como comentábamos, a una cuestión más personal, más particular. Es por ello que tratar respetuosamente a alguien significa «tratarla sobre la base exclusiva de los aspectos de su carácter o sus circunstancias específicas que son efectivamente relevantes para la situación en cuestión»" . Todo lo que conlleve una distorsión de lo que realmente es o acontece a la persona en particular traería aparejada una falta de respeto a la misma: ya sea a causa de promocionarla o impedirla. Es por ello que el tratamiento respetuoso hacia alguien tiene que ver con la «imparcialidad y la evitación de la arbitrariedad» ${ }^{10}$, lo cual pudiera recordarnos, por la forma, al igualitarismo que antes criticaba Frankfurt.

8 Ibid., p. 235.

9 Ibid., p. 235.

10 Ibid, p. 236. 
La cuestión es que, dicha imparcialidad, está orientada a una persona en concreto, la cual es atendida de forma objetiva, pero íntegra y particularmente. Un mero igualitarismo sería una violación de esta atención a la individualidad y, por tanto, una suma falta de respeto. Dicho igualitarismo solo podría darse en casos en los que no pudiésemos conocer información alguna sobre las semejanzas o diferencias entre dos o más personas. En tal caso, igualdad y respeto convergerían por no quedar más remedio, pero solo en un sentido circunstancial y por defecto informativo. Ésta nunca debe ser la postura predeterminada, por ejemplo, en política. Este sentido es importante cuando Frankfurt critica el argumento de Isaiah Berlin por el cual solo la desigualdad requiere razones y no así la igualdad. Al margen del atractivo inicial de esta afirmación, para Frankfurt, sin embargo, se desvela como errónea. Carece de fundamento. La justificación de un trato igualitario vendría dada, como hemos dicho, por un defecto informativo; por una circunstancia fortuita, en definitiva.

La pretensión de igualitarismo estaría fundamentada en un requerimiento más elemental de respeto hacia la persona: ante una circunstancia en la que somos incapaces de establecer alguna comparación entre dos individuos, el respeto a ambos nos hace imposible discriminar a uno frente al otro: finalmente son tratados igualitariamente. Pero esto no significa que se haga por mor de la igualdad, sino del respeto. Es por esto que "lo que determina de manera más decisiva la necesidad de otorgar a todos los seres humanos los mismos derechos es la presunta importancia moral de responder de forma imparcial a su común humanidad, y no la supuesta importancia moral de la igualdad como meta independientemente forzosa» ${ }^{11}$.

Frankfurt se preguntará ahora por qué, entonces, es moralmente imperativo el respeto y por qué es importante guiarnos por lo que de relevante puedan tener las personas. Aquí entrará en juego la racionalidad, entendiendo que guiarse por lo que es relevante constituye, en sí, un elemento imprescindible del ser racional: «la actitud imparcial y respetuosa es en ese sentido un caso especial del hecho del carácter racional $»^{12}$.

De aquí se desprendería fácilmente que el valor moral de este trato a la gente vendría dado, de alguna manera, por el hecho de evitar caer en la irracionalidad que supondría no atender a lo relevante. Es por esto que, si

11 Ibid., p. 238.

12 Ibid., p. 238. 
la importancia de los aspectos relevantes a los que nos atenemos cuando respetamos a alguien nos ha conducido a la racionalidad, dicha racionalidad nos conduce a por qué debiera ser moralmente importante guiarse bajo la misma.

Frankfurt afirmará la deseabilidad natural de guiarse racionalmente para, inmediatamente, pasar a negar cualquier tipo de inmoralidad en la irracionalidad ¿Cómo vinculamos entonces respeto, relevancia, moralidad y racionalidad? Lo que está claro es que las personas que razonan mal no son, por ese único motivo, moralmente culpables: la importancia moral debe buscarse en otra parte.

El lugar donde lo encontramos es, de nuevo, en la persona concreta, en aquella a la que hay que respetar; pero cuando no es respetada. Es en ese maltrato, en ese tratar irrespetuosamente al otro, cuando sale a relucir el carácter moral del respeto: la persona no respetada se siente agraviada, dañada, no tomada su realidad en consideración, o alguna parte de la misma. «La omisión del respeto debido a alguien supone el desconocimiento de la relevancia de algún aspecto de su naturaleza o su situación. [...] En otras palabras, se trata a la persona como si no fuera lo que realmente es» ${ }^{13}$. Aspectos significativos e importantes para la vida de la persona son despreciados y la realidad de la persona negada: en esto consiste la falta de respeto y es por ello que se constituye como moralmente relevante el respetar a los demás, pues evitar dicho daño posee importancia moral. Frankfurt es rotundo al respecto: «Es como si, por negársele el respeto adecuado, se redujera su existencia misma» ${ }^{14}$. Tenemos, por tanto, una buena razón moral para evitar producir esta ofensa y ejercer dicho dańo personal; o, dicho de otra manera, tenemos una buena razón moral para atender a lo vital o existencialmente relevante para otra persona y que, por tanto, nos lleve a tratarla con respeto; conectando, así, moralidad, relevancia, respeto y racionalidad.

Así todo, la gran relevancia moral del respeto, según este enfoque, viene dada como una cuestión de hecho: podemos observar en la práctica los efectos negativos de no tomar en consideración el respeto.

Los elementos que se ponen de relieve en esta exposición son un aparente psicologismo sumado a la atención a las consecuencias, según un criterio

13 Ibid., p. 239.

14 Ibid., p. 239.

Claridades. Revista de filosofía 12/2 (2020) 
que aparece como aparentemente hedonista. De tal manera que primero habría que conocer lo que, subjetivamente, el individuo considera que es relevante dentro de lo que podríamos considerar su biografía existencial y, posteriormente, esto generaría razones para respetarlo atendiendo al posible perjuicio que, de no hacerlo, repercutiría en esta persona.

Este tratamiento de la cuestión que lleva a cabo Frankfurt, aunque audaz, no fue expuesto por el mismo sino como un complemento explicativo (extenso para ser un complemento, pero realmente corto para constituir un ensayo sobre algo como es el respeto) a la cuestión de la igualdad. Sin embargo, llamó poderosamente la atención de Joseph Raz, el cual no pudo resistirse a meditar por sí mismo, y en paralelo diálogo con Frankfurt, lo que dilucidó sobre el respeto. Pasemos a atender, sin más, al tratamiento de la cuestión que Raz llevó brevemente a cabo.

\section{El respeto (II): El ENFOQUe de RaZ}

El comentario de Raz a propósito de la cuestión del respeto suscitada por Frankfurt es realmente más complejo que el que lleva a cabo Frankfurt, pero veamos cómo se va desarrollando dicho comentario para pasar, en último término, a la respuesta dada por el propio Frankfurt.

Raz queda, en alguna medida, encantado con las tesis de Frankfurt, así como con el hecho de que introduzca, en la reflexión filosófica, la cuestión del respeto. Partiendo de varios puntos de acuerdo con el autor (como, por ejemplo, el rechazo por el igualitarismo), Raz se sumará a la pretensión de meditar sobre el respeto a la par que comentará el examen realizado por Frankfurt.

Raz planteará la cuestión preguntándose «si hay alguna razón para respetar a la gente» ${ }^{15}$. Por tanto, es preciso ver si nuestro trato respetuoso (o no) hacia los demás es una respuesta a alguna razón específica respecto a ellos.

Si examinamos, por ejemplo, el gesto de quitarse el sombrero (pasado de moda, por otro lado) a la hora de saludar a otra persona, Raz nos indica que estamos ante un tipo concreto de respeto que podríamos denominar «respeto simbólico». Constituye «acciones simbólicas de las que tenemos

15 Raz, J.: «On Frankfurt's Explanation of Respect for People» en VVAA.: Contours of Agency, ed. Sarah Buss y Lee Overton. Cambridge, Massachusetts: The MIT Press, 2002, p. 299. [NOTA: Toda cita a la obra Contours of Agency es traducción propia]. 
razones para llevarlas a cabo, cuando corresponde, para mostrar nuestro respeto por alguna persona $\mathrm{u}$ institución o causa» ${ }^{16}$. Cuando el respeto es demandado, tenemos, por tanto, razones para respetar y llevar a cabo dichos gestos o símbolos. Pero, nos avisa Raz, este tipo de respeto no es el que tiene la gente en mente. Esto es así porque (1) el sentido de las acciones simbólicas viene dado por la mera tradición o usos sociales y no por provenir de los "preceptos inmutables de la moralidad»; también porque (2) una concepción moral del respeto requiere que respetemos a las personas qua personas, sin entrar en más consideraciones extrínsecas. Necesitamos, por tanto, dar cuenta del respeto al margen de ese respeto simbólico que hemos trazado previamente.

Para Raz, se podría decir que poseemos razones morales respecto al trato a los demás de cuyo cumplimiento podemos derivar, como subproducto [byproduct] el respeto. Es por el correcto ejercicio de nuestras obligaciones morales por lo que podemos decir que actuamos respetuosamente. Pero, si esto es cierto, no cabría decir que hay algo así como razones para respetar como tal.

La interpretación que Raz hace de las tesis de Frankfurt respecto a este punto sería la siguiente: Por un lado «respetar a la gente es meramente un subproducto de actuar por razones invictas [undefeated reasons]. Pero, por otro lado, cuando nuestra acción puede contar como tratar a una persona de una manera o de otra, y nosotros no seguimos las razones invictas, nuestra acción causa un tipo particular de daño que surge del hecho de que la realidad de esa persona es negada ${ }^{17}$. Es de ese daño de donde surgiría una razón independiente para respetar a esa persona. Una razón independiente que nos llevaría a seguir la razón invicta.

Esta postura no respondería a la pretensión de Raz de encontrar una posible independencia del respeto por los demás frente a ese carácter derivado. Raz, entonces, propone cambiar el foco de atención del carácter psicológico, que atribuye a Frankfurt, hacia el normativo. Entonces, lo que importaría no es que una persona, de hecho, se sintiese dañada o su realidad negada cuando no actuamos por razones invictas respecto a cómo tratarla, sino que esa persona tenga derecho a sentir dicho daño. Así, obviar nuestra mejor razón para hacer algo no constituye una falta de respeto.

16 Ibid., p. 300.

17 Ibid., p. 307. 
Sobrevolando ciertos nodos del tratamiento que hace Raz sobre la cuestión respecto a varios matices, podemos establecer su concepción de cómo sería un correcto tratamiento respetuoso hacia los demás basándonos en el valor de dichas personas, quedando así íntimamente relacionadas las nociones de respeto y valor. Una falta de respeto dańaría la realidad del agraviado en el sentido de que no atendería al valor intrínseco de dicha persona. Veámoslo pormenorizadamente:

Podríamos distinguir tres estadios de una respuesta correcta al valor ${ }^{18}$ :

1. En el nivel más básico, hablaríamos de una respuesta psicológicamente apropiada al valor que poseen los objetos a los que atendemos, lo cual incluye emociones, deseos, intenciones, etc. Esto no quiere decir que todo lo que sea considerado de valor lo posea efectivamente. No tenemos más razones para pensar que algo es valioso como para pensar que una proposición es verdadera, pero sí hay una razón general por la cual, si pensamos que algo posee valor, deberíamos de pensar de forma consistente con ese supuesto valor que creemos que posee ${ }^{19}$.

2. "Hay una razón general para preservar lo que es valioso» ${ }^{20}$; si bien es cierto que la fuerza de dicha razón variará en función del valor el objeto. Tenemos, así razones para no destruir lo que consideramos valioso y, por tanto, preservarlo. Las razones para respetar, supone Raz, nos incitan principalmente a no destruir; posiblemente, también a preservar, pero nunca a crear nuevos objetos de valor.

3. Podemos comprometernos con el valor de diversas maneras apropiadas a cada objeto concreto. Así, podemos respetar un libro cuando lo leemos o la música cuando la oímos. Siempre con una actitud y comportamiento apropiado.

Las razones para respetar, son, para Raz, razones que pertenecen a los dos primeros tipos de respuesta al valor; es decir, «razones con respecto a la manera en la que tratamos objetos valiosos en pensamiento y expresión, y razones para preservarlos. Esto difiere de las razones para comprometernos con los objetos valiosos» ${ }^{21}$. Esto es así porque las razones para el respeto son

18 Ibid., pp. 311-12.

19 Ibid., p. 311.

20 Ibid., p. 312.

21 Ibid., p. 313. 
más básicas, más generales, de lo que el compromiso implica. No tiene que ver con gustos o inclinaciones particulares.

Finalmente, de acuerdo con la postura de Raz, «el respeto por las personas es meramente un caso especial de respeto por el valor: respeto por el hecho de que las personas son valiosas» ${ }^{22}$.

\section{El respeto (III): La Respuesta de Frankfurt}

Frankfurt admite que, tal y como Raz observa, su concepción del respeto apunta (y de ahí el interés previo por criticar al igualitarismo) a los comportamientos que se ajustan específicamente a las particularidades de los individuos. Esto, como vimos, no lo acepta Raz, el cual propone una solución basándose en el valor; refiriéndose así, por tanto, a algo más universal y abstracto. Sin embargo, Frankfurt aclara que las particularidades de las que hablaba se refieren a todo lo que uno es: tanto a sus características transitorias y circunstanciales como a aquello relativamente estable de su carácter individual ${ }^{23}$.

Respecto a la cuestión de la racionalidad, según Frankfurt, entendimos que tratar a la gente con respeto formaba parte de lo que significa ser racional, y esto es así porque es propiamente irracional violar el principio según el cual la conducta debe guiarse por lo que es relevante. Evitar un daño a la persona a la que debemos respetar constituye, por tanto, una razón moral pertinente para tratarla con dicho respeto. El problema viene en tanto que, para Frankfurt, Raz le está atribuyendo el hecho de que respetamos a los demás cuando las tratamos por buenas razones invictas, mientras que, según Frankfurt, él nunca ha sostenido tal cosa. Para Frankfurt, tratar irrespetuosamente a alguien implica algo mucho más concreto que el requerimiento de racionalidad o ignorar buenas razones invictas. Implica actuar de tal manera que se niegue la realidad de la persona.

Por otro lado, Frankfurt no considera, a diferencia de Raz, que el respeto esté completamente separado de los motivos y creencias (es decir, del aspecto psicológico del que le acusa Raz). Esto lo ilustra perfectamente el ejemplo que pone Raz del chico que se levanta para hablar ante el profesor. Para Raz, ese chico expresa respeto aun si él no es consciente de ello.

22 Ibid., p. 313.

23 Frankfurt, H.G.: «Reply to Joseph Raz» en VVAA.: Contours of Agency, ed. Sarah Buss y Lee Overton. Cambridge, Massachusetts: The MIT Press, 2002, p. 316. 
Frankfurt contraargumenta que, si el alumno viene de una sociedad en la que ese gesto constituye una expresión de desafío, en la sociedad en la que es considerado como un gesto de respeto seguiría siendo, sin embargo, un acto irrespetuoso, pues la intención del alumno era la de desafiar y contravenir. Así, podríamos entender que, para Frankfurt, Raz se equivoca al desvincular el respeto de las creencias y deseos.

Es relevante tomar lo último en consideración. Y para ello nos puede servir traer a colación la distinción kantiana entre actuar conforme al deber o por deber, transformada ahora en la distinción actuar conforme al respeto (pretendido) o por respeto. Tal y como reformula Frankfurt la situación del alumno y el profesor, queda patente que el alumno actúa conforme al respeto socialmente esperable (aun sin saberlo), pero no por respeto.

Es en este punto donde se reivindica con especial fuerza eso que Raz acusa de psicologismo. Realmente no podemos considerar si alguien está siendo respetuoso no si no tenemos mínimamente en consideración, aunque sea de forma tácita, la subjetividad de quien se supone que debe respetar ¿Cuántas veces alguien ha dudado de que se le esté respetando de verdad, aun cuando externamente se cumplan los requisito aparentes? Nos importa sentir que la gente nos respeta en conciencia, más allá de lo que podríamos denominar, ser educados o educadas.

En último lugar, la diferencia más significativa entre ambos autores viene de la relación entre respeto y valor. Así, Frankfurt se niega rotundamente a entender que no respetar a alguien consista en un acto que niegue el valor intrínseco de todas las personas. Frankfurt afirma que no hay ninguna relación filosóficamente relevante entre respeto y valor (intrínseco a la persona, y no de otro posible tipo). Y es que, si algo no posee valor para nosotros, podemos deshacernos de ello sin perder nada. En cambio, si para no respetar a una persona hemos de hacer lo mismo, entonces no estaríamos sino incurriendo en la «más extraordinariamente irrespetuosa de las acciones ${ }^{24}$, lo cual es esclarecedor para percibir la distinción.

En este punto, el concepto de respeto comparte tangencialmente un aspecto similar al de la tolerancia: no tenemos que esperar a compartir (o en este caso valorar) algo de alguien para tolerarlo o respetarlo. Ciertamente, en el caso de la tolerancia es requisito no estar de acuerdo para que sea un acto original de tolerancia. El respeto, por su lado, simplemente puede y

24 Ibid., p. 319. 
sería bueno que se diese, al margen de que tengamos que valorar algo de alguien. Más bien consideramos la importancia que aquello tiene para la persona que respetamos.

Para Frankfurt, la manera en la que Raz entiende el valor personal es algo distinta. Esto es así porque no hay ninguna necesidad en entender que, si no respetamos a alguien, lo estemos tratando como un medio en vez de como un fin. Por tanto, el enfoque de Frankfurt desvincula el respeto de cualquier consideración respecto al supuesto valor personal de lo respetado. $\mathrm{Y}$ es que se puede faltar al respeto perfectamente obviando, de hecho, aspectos no valiosos de la persona. Un ejemplo de esto sería aquel en el que se realiza una lista de personas de cierta estatura y se obvia a alguien de dicha estatura. Se ha obviado un aspecto de la realidad de dicha persona, por lo que no se la ha respetado, pese a que era un aspecto carente de valor por sí mismo. Lo importante, sería, por tanto, lo característico y conformante de la realidad del individuo y no su supuesto valor inherente.

\section{Conclusiones}

El tratamiento analítico que dos autores de esta talla han otorgado al concepto de respeto debe de ser tenido en cuenta para futuros abordajes. Raz, pese a todo, no hace, sino mantener una referencia ya clásica y común a la dignidad (no mencionada, pero sí detectable, a mi juicio) como garante del respeto a las personas en tanto que valiosas en sí mismas. Frankfurt, sin embargo, no echa mano de ninguna consideración igualitaria o entidad metafísica velada, sino que ofrece una alternativa sencilla y natural a por qué es moralmente relevante respetar a los demás en términos de los perjuicios que una omisión de dicho respeto acarrearía. Así todo, entiendo que respetar a alguien consistiría, de alguna u otra forma, en tener en consideración a la otra persona. Prima, al menos para Frankfurt, la atención a la diferenciación constitutiva de la persona concreta frente a una igualdad genérica, y en teoría valiosa, de la persona abstracta por la que aboga Raz.

Ciertamente, cabe pensar que, en el marco establecido por Frankfurt, el carácter relevante de los diversos aspectos diferenciadores de la persona alude, de alguna manera, a una valoración. Sin embargo, es señalable que esto no remite al valor como meollo del respeto (postura que sostendría Raz). Los valores que ponemos en juego son distintos. El valor, en la postura de Raz, apela a un valor intrínseco de la persona en su totalidad: se 
respeta "por el hecho de que las personas son valiosas ${ }^{25}$ »; es decir, la persona es valiosa en sí misma, por el mero hecho de ser persona. La similitud con la dignidad es elevada.

Por otro lado, si interpretásemos un posible valor en el planteamiento de Frankfurt, deberíamos aludir a un valor que el individuo atribuiría a algún aspecto propio y relevante para él en algún contexto dado. Sin embargo, no es preciso acudir al valor como clave para la comprensión del respeto. Así, se me faltaría al respeto, como comentábamos, si no se tiene en cuenta mi estatura, por ejemplo, al examinarme para las oposiciones a un cuerpo del Estado que requiera una estatura mínima, que yo excedo. Medir más o menos no es una cuestión intrínsecamente valiosa (aunque yo pudiera concederle valor; lo cual ya constituye un valor distinto al que reivindica Raz), sino que constituye un aspecto diferencial de la persona que, en un contexto dado, debe ser tenido en cuenta. Lo contrario, produce un agravio, el cual justifica la incorrección moral de la falta de respeto. El perjuicio, en este caso, no viene dado porque la persona no haya sido valorada, sino porque no ha sido tenida en cuenta una faceta propia relevante (no valiosa en un sentido intrínseco, sino pertinente y conformadora de los rasgos que caracterizan a dicho individuo).

De la misma manera, si insistiésemos en la necesidad de concederle valor a dichas características relevantes, cabría preguntarse por qué le concedemos tal valor. La respuesta vendría dada por el hecho de que constituyen, precisamente, rasgos diferenciadores que exigen ser tenidos en cuenta. En el peor de los casos, caeríamos en una suerte de circularidad por la que valor y diferencia se referenciarían mutuamente: el aspecto diferencial es relevante porque es valioso y es valioso precisamente porque constituye un rasgo propio a ser tenido en cuenta en X circunstancia que, de no ser así, causaría un perjuicio.

Más arriba señalamos la caracterización que Raz hizo de Frankfurt como psicologista, a la vez que indicamos la necesidad de atender a las razones, creencias y deseos para con la propia biografía existencial con vistas a poder entender si alguien es de hecho respetuoso o no. Poro otro lado, vemos que tal psicologismo es excesivo y no es fiel a todo el enfoque frankfurtiano:

25 Raz, J.: «On Frankfurt's Explanation of Respect for People» en VVAA.: Contours of Agency, ed. Sarah Buss y Lee Overton. Cambridge, Massachusetts: The MIT Press, 2002, p. 313. 
vimos que el contexto establece un criterio de pertinencia de los aspectos relevantes o no a respetar. Es por ello que constituye una visión integradora del respeto, donde lo objetivo y lo subjetivo son tomados en consideración.

Esta dualidad implica un movimiento dialéctico en el que se hace preciso el entendimiento entre lo social y lo individual. Lo social no puede excederse en sus pretensiones homogeneizadoras irrespetando lo diferente y moralmente valioso del individuo concreto. Así mismo, la comunidad y su lugares establecen criterios externos que ponen en valor o no distintas facetas de lo humano a considerar. Haciendo superfluas ciertas exigencias de respeto o, por el contrario, reivindicando otras. En este juego, que es la vida misma, es preciso el entendimiento y la apertura a los demás.

\section{REFERENCIAS BIBLIOGRÁFICAS.}

Frankfurt, Harry G. «Igualdad y respeto». En Necesidad, volición y amor. Buenos Aires: Katz, 2007.

Frankfurt, Harry G. «La igualdad como ideal moral». En La importancia de lo que nos preocupa. Buenos Aires: Katz, 2006.

Frankfurt, Harry G. «Reply to Joseph Raz». En Contours of Agency, editado por Sarah Buss y Lee Overton. Cambridge, Massachusetts: The MIT Press, 2002.

Raz, Joseph. «On Frankfurt's Explanation of Respect for People». En Contours of Agency, editado por Sarah Buss y Lee Overton. Cambridge, Massachusetts: The MIT Press, 2002.

Gabriel Aragón Aranda es es profesor de Filosofía en la educación secundaria y doctorando en Ciencias de la Educación.

Lineas de investigación:

Didáctica de la Filosofía.

Videojuegos y Educación.

\section{Publicaciones recientes:}

- «Contra el utilitarismo: el heterogéneo enfoque de los teóricos de la virtud» en Claridades 9, 2017, pp. 67-88.

Dirección electrónica: gabriel.aragon@uma.es 